\title{
Relativistic Spin-0 Particle Under Linear Central Potential Effects Induced by Lorentz Symmetry Violation
}

\author{
Faizuddin Ahmed ${ }^{1}$ \\ National Academy Gauripur, Assam, 78331, India
}

\begin{abstract}
In this work, we investigate the behaviour of a relativistic scalar particle in the background of the Lorentz symmetry violation determined by a tensor $\left(K_{F}\right)_{\mu \nu \alpha \beta}$ out of the Standard Model Extension. A linear electric field and a uniform magnetic can be induced by the violation of the Lorentz symmetry breaking effects, and analyze the behaviour of the scalar particle. We see that the analytical solution to the KG-equation can be achieved, and a quantum effect characterized by the dependence of the magnetic field on the quantum numbers is observed.
\end{abstract}

Keywords: Lorentz symmetry violation, Relativistic wave-equations, electric \& magnetic field, biconfluent Heun's function, special function .

PACS Number(s): 11.30.Qc, 11.30.Cp, 03.65.Pm,

\section{Introduction}

Recent studies have investigated the possibility of Lorentz-symmetry violation from the generalization of the usual standard model called the standardmodel extension(SME), that has all the conventional properties but breaks the Lorentz-symmetry $[1,2,3,4]$. This framework provides a quantitative description of Lorentz-symmetry violation, controlled by a set of coefficients

\footnotetext{
${ }^{1}$ faizuddinahmed15@gmail.com ; faiz4U.enter@rediffmail.com
} 
whose values are to be determined or constrained by experiments $[5,6,7,8]$. In this model, the CPT- and Lorentz-breakdown take place as a spontaneous violation $[9,10,11]$ in the context of more fundamental theory, valid at the Planck scale. The parameters representing Lorentz violation are obtained as vacuum expectation values of some tensor operators belonging to the underlying theory. The gauge sector of the SME model has been extensively studied in several works both in $(1+3)$ and $(1+2)$-dimensions $[12,13,14,15,16,17,18,19,20,21,22,23,24,25,26,27,28,29$, 30], with many interesting results.

We study the relativistic quantum dynamics of a scalar particle Refs. $[31,32,33]$ by solving the Klein-Gordon equation under the effects of violation of the Lorentz symmetry defined by the tensor $\left(K_{F}\right)_{\mu \nu \alpha \beta}$ out of the Standard Model Extension which hasn't studied earlier. We investigate the effects of linear central potential induced by Lorentz symmetry violation and obtain the solution of the bound state.

\section{Relativistic scalar particle under the effects of Lorentz Symmetry Violation}

The gauge sector of the Standard Model Extension consists two violating terms that modifies the transport properties of space-time since these terms break the Lorentz symmetry. These two terms are called the CPT-odd sector $[1,2]$ and the CPT-even sector $[34,35]$. The relativistic quantum dynamics of a scalar particle under the effects of the Lorentz symmetry violation $[1,2$, $3,36,37,38,39,40,41,42,43,44,45]$

$$
p^{\mu} p_{\mu} \Psi+\frac{\alpha}{4}\left(K_{F}\right)_{\mu \nu \alpha \beta} F^{\mu \nu}(x) F^{\alpha \beta}(x) \Psi=M^{2} \Psi
$$

where $\alpha$ is a constant, $F_{\mu \nu}(x)=\partial_{\mu} A_{\nu}-\partial_{\nu} A_{\mu}$ is the electromagnetic tensor, $\left(K_{F}\right)_{\mu \nu \alpha \beta}$ corresponds to a tensor that governs the Lorentz symmetry violation out of the Standard Model Extension. One of the properties of 
the tensor $\left(K_{F}\right)_{\mu \nu \alpha \beta}$ is that it can be written in terms of $3 \times 3$ matrices: $\left(\kappa_{D E}\right)_{j k}=-2\left(K_{F}\right)_{0 j 0 k}$ and $\left(\kappa_{H B}\right)_{j k}=\frac{1}{2} \epsilon_{j p q} \epsilon_{k l m}\left(K_{F}\right)^{p q l m}$ representing the parity-even sector, and others are represented by $3 \times 3$ matrices as : $\left(\kappa_{D B}\right)_{j k}=-\left(\kappa_{H E}\right)_{k j}=\epsilon_{k p q}\left(K_{F}\right)^{0 j p q}$ representing parity-odd sector. Note that the matrices $\left(\kappa_{D E}\right)_{j k}$ and $\left(\kappa_{H B}\right)_{j k}$ are symmetric and the matrices $\left(\kappa_{D B}\right)_{j k}$ and $\left(\kappa_{H E}\right)_{k j}$ have no symmetry.

The KG-equation (1) under the effects of the Lorentz symmetry violation in the Minkowski space-time background becomes

$$
\begin{aligned}
& {\left[-\frac{\partial^{2}}{\partial t^{2}}+\frac{\partial^{2}}{\partial r^{2}}+\frac{1}{r} \frac{\partial}{\partial r}+\frac{\partial^{2}}{\partial z^{2}}+\frac{1}{r^{2}} \frac{\partial^{2}}{\partial \phi^{2}}\right] \Psi+\frac{\alpha}{4}\left(K_{F}\right)_{\mu \nu \alpha \beta} F^{\mu \nu}(x) F^{\alpha \beta}(x) \Psi} \\
& =M^{2} \Psi
\end{aligned}
$$

Using the properties of the tensor field $\left(K_{F}\right)_{\mu \nu \alpha \beta}$, we rewrite (2) in the form :

$$
\begin{aligned}
& {\left[-\frac{\partial^{2}}{\partial t^{2}}+\frac{\partial^{2}}{\partial r^{2}}+\frac{1}{r} \frac{\partial}{\partial r}+\frac{1}{r^{2}} \frac{\partial^{2}}{\partial \phi^{2}}+\frac{\partial^{2}}{\partial z^{2}}\right] \Psi} \\
& +\left[-\frac{\alpha}{2}\left(\kappa_{D E}\right)_{i j} E^{i} E^{j}+\frac{\alpha}{2}\left(\kappa_{H B}\right)_{j k} B^{i} B^{j}-\alpha\left(\kappa_{D B}\right)_{j k} E^{i} B^{j}\right] \Psi \\
& =M^{2} \Psi
\end{aligned}
$$

Let us consider a possible scenario of the Lorentz symmetry violation determined by $\left(\kappa_{D E}\right)_{11}=$ const, $\left(\kappa_{H B}\right)_{33}=$ const and $\left(\kappa_{D B}\right)_{13}=$ const and the field configuration given by $[30,39,40,28,29,46]$ :

$$
\vec{B}=B_{0} \hat{z} \quad, \quad \vec{E}=\frac{\lambda}{2} r \hat{r}
$$

where $B_{0}>0, \hat{z}$ is a unit vector in the $z$-direction, $\lambda$ is a constant associated with a linear distribution of electric charge along the axial direction, and $\hat{r}$ is the unit vectors in the radial direction.

Hence, the equation (3) using the configuration (4) becomes

$$
\begin{aligned}
& {\left[-\frac{\partial^{2}}{\partial t^{2}}+\frac{\partial^{2}}{\partial r^{2}}+\frac{1}{r} \frac{\partial}{\partial r}+\frac{1}{r^{2}} \frac{\partial^{2}}{\partial \phi^{2}}+\frac{\partial^{2}}{\partial z^{2}}\right] \Psi} \\
& +\left[-\frac{\alpha \lambda^{2}}{8}\left(\kappa_{D E}\right)_{11} r^{2}+\frac{\alpha}{2}\left(\kappa_{H B}\right)_{33} B_{0}^{2}-\frac{\alpha \lambda B_{0}}{2}\left(\kappa_{D B}\right)_{13} r\right] \Psi=M^{2} \Psi .(5)
\end{aligned}
$$


Since the metric is independent of time and symmetrical by translations along the $z$-axis, as well by rotations. It is reasonable to write the solution to Eq. (6) as

$$
\Psi(t, r, \phi, z)=e^{i(-E t+l \phi+k z)} \psi(r),
$$

where $E$ is the energy of the particle, $l=0, \pm 1, \pm 2, \ldots$ are the eigenvalues of the $z$-component of the angular momentum operator, and $k$ is a constant.

Substituting the solution (6) into the Eq. (7), we obtain the following radial wave-equation for $\psi(r)$ :

$$
\psi^{\prime \prime}(r)+\frac{1}{r} \psi^{\prime}(r)+\left[\Lambda-\omega^{2} r^{2}-\frac{l^{2}}{r^{2}}-b r\right] \psi(r)=0,
$$

where

$$
\begin{aligned}
& \Lambda=E^{2}-M^{2}-k^{2}+\frac{1}{2} \alpha B_{0}^{2}\left(\kappa_{H B}\right)_{33}, \\
& \omega=\sqrt{\frac{1}{8} \alpha \lambda^{2}\left(\kappa_{D E}\right)_{11}}>0, \\
& b=\frac{1}{2} \alpha \lambda B_{0}\left(\kappa_{D B}\right)_{13} .
\end{aligned}
$$

Let us perform a change of variables given by $x=\sqrt{\omega} r$. Then, we have

$$
\psi^{\prime \prime}(x)+\frac{1}{x} \psi^{\prime}(x)+\left[\zeta-x^{2}-\frac{l^{2}}{x^{2}}-\theta x\right] \psi(x)=0,
$$

where

$$
\zeta=\frac{\Lambda}{\omega} \quad, \quad \theta=\frac{b}{\omega^{\frac{3}{2}}} .
$$

By analysing the asymptotic behaviour of Eq. (9) at $x \rightarrow 0$ and $x \rightarrow \infty$, we have a solution to Eq. (9) that can be written in terms of an unknown function $F(x)$ as

$$
\psi(x)=x^{|l|} e^{-\frac{1}{2}(x+\theta) x} H(x)
$$

Thereby, substituting Eq. (11) into Eq. (9), we obtain

$$
H^{\prime \prime}(x)+\left[\frac{1+2|l|}{x}-2 x-\theta\right] H^{\prime}(x)+\left[-\frac{\frac{\theta}{2}(1+2|l|)}{x}+\Theta\right] H(x)=0,
$$


where $\Theta=\zeta+\frac{\theta^{2}}{4}-2(1+|l|)$.

The equation (12) is the biconfluent Heun's differential equation [31, 32, $33,47,48,49]$ with $H(x)$ is the Heun polynomials function.

The above equation (12) can be solved by the Frobenius method. Writing the solution as a power series expansion around the origin [50]:

$$
H(x)=\sum_{i=0}^{\infty} d_{i} x^{i}
$$

Substituting the power series solution into the Eq. (12), we obtain the following recurrence relation

$$
d_{n+2}=\frac{1}{(n+2)(n+2+2|l|)}\left[\theta\left(n+|l|+\frac{3}{2}\right) d_{n+1}-(\Theta-2 n) d_{n}\right] .
$$

With few coefficients are

$$
\begin{aligned}
& d_{1}=\frac{\theta}{2} d_{0}, \\
& d_{2}=\frac{1}{4(1+|l|)}\left[\theta\left(|l|+\frac{3}{2}\right) d_{1}-\Theta d_{0}\right] .
\end{aligned}
$$

The power series expansion $H(x)$ becomes a polynomial of degree $n$ by imposing the following two conditions [31, 32, 33, 30, 39, 28, 29, 47]

$$
\begin{aligned}
\Theta & =2 n, \quad(n=1,2, \ldots) \\
d_{n+1} & =0 .
\end{aligned}
$$

By analysing the first condition $\Theta=2 n$, we have

$$
E_{n, l}= \pm \sqrt{M^{2}+k^{2}+(n+1+|l|) \lambda \sqrt{\frac{\alpha}{2}\left(\kappa_{D E}\right)_{11}}-\frac{\alpha \chi B_{0}^{2}}{2}}
$$

where $\chi=\left[\left(\kappa_{H B}\right)_{33}+\frac{\left\{\left(\kappa_{D B}\right)_{13}\right\}^{2}}{\left(\kappa_{D E}\right)_{11}}\right]$.

The equation (17) gives the allowed values of the energy eigenvalue of a relativistic scalar particle under the effects of a linear central potential induced by Lorentz symmetry breaking out of the Standard Model Extension. 
In the present situation, Lorentz symmetry breaking effects defined by a tensor that governs the Lorentz symmetry violation have non-null components $\left(\kappa_{D B}\right)_{13}=$ const, $\left(\kappa_{D E}\right)_{11}=$ const and $\left(\kappa_{H B}\right)_{33}=$ const, a linear radial electric field produced by linear distribution of electric charges, and a uniform magnetic along the $z$-direction. Note that Eq. (17) is not the general expression of the energy eigenvalues. One can obtain the individual energy levels $E_{1, l}, E_{2, l}, E_{3, l}, \ldots$ and the wave-function $\psi_{1, l}, \psi_{2, l}, \ldots \ldots$ for each radial mode $n=1,2,3, \ldots$ by imposing the additional recurrence condition $d_{n+1}=0$ on the eigenvalue problem as done in Refs. [31, 32, 33, 47].

For the radial mode $n=1$, we have $\Theta=2$ and $d_{1}=0$ which implies from (15)

$$
\begin{aligned}
& \frac{\theta}{2} d_{0}=\frac{2}{\theta\left(|l|+\frac{3}{2}\right)} d_{0} \\
\Rightarrow & \frac{b^{2}}{4}=\frac{\omega^{3}}{\left(\frac{3}{2}+|l|\right)} \Rightarrow \lambda_{1, l}=\left(\frac{3}{2}+|l|\right)\left(\kappa_{D B}\right)_{13} B_{0}^{2} \sqrt{\frac{2 \alpha}{\left\{\left(\kappa_{D E}\right)_{11}\right\}^{3}}}
\end{aligned}
$$

a constraint on the linear charge density $\lambda_{1, l}$ for the radial mode $n=1$. We can see that for the different quantum numbers of the system, there is a different relation of the linear charge density $\lambda$, so we have labelled $\lambda \rightarrow \lambda_{n, l}$. Note that its values depends on the Lorentz symmetry violation parameters $\left\{\left(\kappa_{D B}\right)_{13},\left(\kappa_{D E}\right)_{11}\right\}$ as well as on the quantum numbers $\{n, l\}$ of the relativistic system which shows a quantum effect.

Therefore, the lowest state energy level for the radial mode $n=1$ is

$$
\begin{aligned}
E_{1, l} & = \pm \sqrt{M^{2}+k^{2}+(2+|l|) \lambda_{1, l} \sqrt{\frac{\alpha}{2}\left(\kappa_{D E}\right)_{11}}-\frac{\alpha \chi B_{0}^{2}}{2}} \\
& =\sqrt{M^{2}+k^{2}+\frac{1}{2} \alpha B_{0}^{2}\left\{(2+|l|)(3+2|l|) \frac{\left\{\left(\kappa_{D B}\right)_{13}\right\}^{2}}{\left(\kappa_{D E}\right)_{11}}-\chi\right\}} \cdot(1)
\end{aligned}
$$

The corresponding ground state wave-function is

$$
\psi_{1, l}(x)=x^{|l|} e^{-\left(\frac{x}{2}+d_{1}\right) x}\left(1+d_{1} x\right)
$$


where we have chosen $d_{0}=1$ and

$$
d_{1}=\frac{1}{\sqrt{\frac{3}{2}+|l|}} .
$$

We can see that the presence of Lorentz symmetry breaking parameters modified the energy level and the wave-function of a relativistic scalar particle in the quantum system.

\section{Special case corresponds to $\left(\kappa_{D B}\right)_{13}=0$}

Here we shall discuss the above relativistic quantum system corresponds to the non-null components $\left(\kappa_{D E}\right)_{11}=$ const and $\left(\kappa_{H B}\right)_{33}=$ const of the tensor that governs the Lorentz symmetry breaking effects and other is zero. In that case, the radial wave-equation (7) becomes

$$
\psi^{\prime \prime}(r)+\frac{1}{r} \psi^{\prime}(r)+\left(\Lambda-\omega^{2} r^{2}-\frac{l^{2}}{r^{2}}\right) \psi(r)=0,
$$

Transforming $\rho=\omega r^{2}$ into the Eq. (22), we have

$$
\psi^{\prime \prime}(\rho)+\frac{1}{\rho} \psi^{\prime}(\rho)+\frac{1}{4 \rho}\left(\frac{\Lambda}{\omega}-\rho-\frac{l^{2}}{\rho}\right) \psi(\rho)=0,
$$

The above equation can be transform to the following differential equation $[51,52,53]$

$$
\psi^{\prime \prime}(\rho)+\frac{1}{\rho} \psi^{\prime}(\rho)+\frac{1}{\rho^{2}}\left(-\xi_{1} \rho^{2}+\xi_{2} \rho-\xi_{3}\right) \psi(\rho)=0,
$$

where

$$
\xi_{1}=\frac{1}{4} \quad, \quad \xi_{2}=\frac{\Lambda}{4 \omega} \quad, \quad \xi_{3}=\frac{l^{2}}{4} .
$$

Comparing Eq. (24) with Eq. (A.1) in Ref. [53]

$$
\begin{aligned}
& \alpha_{1}=1 \quad, \quad \alpha_{2}=0 \quad, \quad \alpha_{3}=0 \quad, \quad \alpha_{4}=0 \quad, \quad \alpha_{5}=0, \\
& \alpha_{6}=\xi_{1} \quad, \quad \alpha_{7}=-\xi_{2} \quad, \quad \alpha_{8}=\xi_{3} \quad, \quad \alpha_{9}=\xi_{1} \quad, \quad \alpha_{10}=1+2 \sqrt{\xi_{3}}, \\
& \alpha_{11}=2 \sqrt{\xi_{1}} \quad, \quad \alpha_{12}=\sqrt{\xi_{3}} \quad, \quad \alpha_{13}=-\sqrt{\xi_{1}} .
\end{aligned}
$$


The eigenvalues equation using Eqs. (25)-(26) into the Eq. (A.8) in Ref. $[53]$

$$
\begin{aligned}
E_{n^{\prime}, l} & = \pm \sqrt{M^{2}+k^{2}+2 \omega\left(2 n^{\prime}+1+|l|\right)-\frac{1}{2} \alpha B_{0}\left(\kappa_{H B}\right)_{33}} \\
& = \pm \sqrt{M^{2}+k^{2}+\lambda\left(2 n^{\prime}+1+|l|\right) \sqrt{\frac{\alpha}{2}\left(\kappa_{D E}\right)_{11}}-\frac{1}{2} \alpha B_{0}\left(\kappa_{H B}\right)_{33}}
\end{aligned}
$$

where $n^{\prime}=0,1,2,3 \ldots \ldots$

The normalized radial wave function is given by

$$
\psi_{n^{\prime}, l}=|N|_{n^{\prime}, l} \rho^{\frac{|l|}{2}} e^{-\frac{\rho}{2}} L_{n^{\prime}}^{(|l|)}(\rho)
$$

where $|N|_{n^{\prime}, l}=\left(\frac{n^{\prime} !}{\left(n^{\prime}+|l|\right) !}\right)^{\frac{1}{2}}$ is the normalization constant and $L_{n^{\prime}}^{(|l|)}(\rho)$ is the generalized Laguerre polynomials and are orthogonal over $(0, \infty]$ with respect to the measure with weighting function $\rho^{|l|} e^{-\rho}$ as

$$
\int_{0}^{\infty} \rho^{|l|} e^{-\rho} L_{n^{\prime}}^{(|l|)}(\rho) L_{m^{\prime}}^{(|l|)}(\rho) d \rho=\left(\frac{\left(n^{\prime}+|l|\right) !}{n^{\prime} !}\right) \delta_{n^{\prime} m^{\prime}} .
$$

We see from Eqs. (27)-(30) that the energy eigenvalue and the normalized eigenfunction which depend on the Lorentz symmetry breaking parameters get modified.

For zero Lorentz symmetry parameter $\left(\kappa_{H B}\right)_{33}=0$, the energy eigenvalues (27) becomes

$$
E_{n^{\prime}, l}= \pm \sqrt{M^{2}+k^{2}+\lambda\left(2 n^{\prime}+1+|l|\right) \sqrt{\frac{\alpha}{2}\left(\kappa_{D E}\right)_{11}}}
$$

with the same normalized radial wave-function (28).

\section{Conclusions}

In this work, we have studied the effects of central potential induced by the violation of the Lorentz symmetry on a relativistic scalar particle. The 
central potential is chosen by a linear radial electric field produced by a linear distribution of electric charges, a uniform magnetic field along the $z$ direction, and the tensor field $\left(K_{F}\right)_{\mu \nu \alpha \beta}$ that governs the Lorentz symmetry violation out of the SME that possesses the non-null components $\left(\kappa_{D B}\right)_{13}=$ const, $\left(\kappa_{D E}\right)_{11}=$ const and $\left(\kappa_{H B}\right)_{33}=$ const. We have derived the radial wave equation and for a suitable function, the biconfluent Heun differential equation for is arrived. Using a power series solution in this Heun equation and finally truncating the series solution of $H(x)$ to a finite degree polynomial such that the wave function $\psi(x)$ must be well-behaved in the limit $x \rightarrow 0$ and $x \rightarrow \infty$. By analysing the truncating conditions, one can obtained the energy eigenvalues Eq. (13) and the radial wave function Eq. (14) of a relativistic scalar particle. Since the energy eigenvalues (13) is not the general expression, by imposing the recurrence condition $d_{n+1}=0$, one can obtain the individual energy level $E_{1}, E_{2}, E_{3}, .$. and the wave function $\psi_{1, l}, \psi_{2, l}, \psi_{3, l}, \ldots$ for the radial mode $n=1,2,3, \ldots$. As for example, the lowest state energy level Eq. (19) and the wave function Eqs. (20)-(21) with the restriction on the linear charge density $\lambda_{1, l}$ Eq. (18) for the radial mode $n=1$ is obtained. We have seen a quantum effect due to the dependence of the linear charge density on the quantum numbers $\{n, l\}$ of the relativistic system as well as on the Lorentz symmetry parameters. We also see that the presence of the Lorentz symmetry breaking parameters modified the energy spectrum and wave function. Further, we have discussed a special case corresponding to zero Lorentz symmetry parameter $\left(\kappa_{D B}\right)_{13}=0$ and others are non-null, and arrived the second order differential radial wave equation. Using the Nikiforov-Uvarov method, we have obtained the energy eigenvalues Eq. (27) and the normalized wave function (28).

\section{Conflict of Interest}

There is no conflict of interests regarding publication this manuscript. 


\section{Data Availability}

There is no data associated with this manuscript.

\section{References}

[1] D. Colladay and V. A. Kostelecky, Phys. Rev. D 55, 6760 (1997).

[2] D. Colladay and V. A. Kostelecky, Phys. Rev. D 58, 116002 (1998).

[3] D. Colladay and V. A. Kostelecky, Phys. Lett. B 511, 209 (2001).

[4] V. A. Kostelecky and R. Lehnert, Phys. Rev. D 63, 065008 (2001).

[5] L. B. Auerbach et. al., (The LSND Collaboration), Phys. Rev. D 72, 076004 (2005).

[6] Y. B. Hsiung et. al. (The KTeV Collaboration), Nucl. Phys. Proc. Suppl. 86, $312(2000)$.

[7] H. Dehmelt, R. Mittleman, R. S. Van Dyck, Jr. and P. Schwinberg, Phys. Rev. Lett. 83, 4694 (1999).

[8] B. Heckel, C. E. Cramer, T. S. Cook, E. G. Adelberger, S. Schlamminger and U. Schmidt, Phys. Rev. Lett. 97, 021603 (2006).

[9] V. A. Kostelecky and S. Samuel, Phys. Rev. D 39, 683 (1989).

[10] V. A. Kostelecky and R. Potting, Nucl. Phys. B 359, 545 (1991).

[11] V. A. Kostelecky and R. Potting, Phys. Rev. D 51, 3923 (1995).

[12] 4. S. R. Coleman and S. L. Glashow, Phys. Rev. D 59, 116008 (1999).

[13] J. Lipa, J. A. Nissen, S. Wang, D. A. Stricker, and D. Avaloff, Phys. Rev. Lett. 90, 060403 (2003). 
[14] H. Muller, S. Herrmann, C. Braxmaier, S. Schiller and A. Peters, Phys. Rev. Lett. 91, 020401 (2003).

[15] M. Frank and I. Turan, Phys. Rev. D 74, 033016 (2006).

[16] 5. C. Adam and F. R. Klinkhamer, Nucl. Phys. B 607, 247 (2001).

[17] 5. C. Adam and F. R. Klinkhamer, Nucl. Phys. B 657, 214 (2003).

[18] R. Montemayor and L. F. Urrutia, Phys. Rev. D 72, 045018 (2005).

[19] R. Lehnert, Phys. Rev. D 68, 085003 (2003).

[20] R. Lehnert, Int. J. Mod. Phys. A 20, 1303 (2005).

[21] H. Belich, M. M. Ferreira Jr., J. A. Helayel-Neto and M. T. D. Orlando, Phys. Rev. D 67, 125011 (2003).

[22] H. Belich, M. M. Ferreira Jr., J. A. Helayel-Neto and M. T. D. Orlando, Phys. Rev. D 69, 109903 (2004).

[23] H. Belich, M. M. Ferreira Jr., J. A. Helayel-Neto and M. T. D. Orlando, Phys. Rev. D 68, 025005 (2003).

[24] H. Belich, J. L. Boldo, L. P. Colatto, J. A. Helayël-Neto and A. L. M. A. Nogueira, Phys. Rev. D 68, 065030 (2003).

[25] H. Belich, M. M. Ferreira Jr, and J. A. Helayel-Neto, Eur. Phys. J. C 38, 511 (2005).

[26] H. Belich Jr., T. Costa-Soares, M. M. Ferreira Jr. and J. A. Helayel-Neto, Eur. Phys. J. C 42, 127 (2005).

[27] H. Belich Jr., T. Costa-Soares, M. M. Ferreira Jr. and J. A. Helayel-Neto, Eur. Phys. J. C 41, 421 (2005).

[28] M. Ericsson and E. Sjoqvist, Phys. Rev. A 65, 013607 (2001). 
[29] I. C. Fonseca and K. Bakke, J. Math. Phys. 56, 062107 (2015).

[30] K. Bakke and H. Belich, Ann. Phys. (N. Y) 354, 1 (2015).

[31] F. Ahmed, Eur. Phys. J. C 78, 598 (2018).

[32] E. R. F. Medeiros and E. R. Bezerra de Mello, Eur. Phys. J. C 72, 2051 (2012).

[33] H. Sobhani, H. Hassanabadi and W. S. Chung, Int. J. Geom. Meths Mod. Phys. 15 (03), 1850037 (2018).

[34] S. Carroll, G. Field, R. Jackiw, Phys. Rev. D 41, 1231 (1990).

[35] A. P. Baeta Scarpelli, H. Belich, J. L. Boldo, L. P. Colatto, J. A. HelayelNeto, A. L. M. A. Nogueira, Nucl. Phys. (Proc. Suppl.) 127, 105 (2004).

[36] V. A. Kostelecky and M. Mewes, Phys. Rev. Lett. 87, 251304 (2001).

[37] V. A. Kostelecky and M. Mewes, Phys. Rev. D 66, 056005 (2002).

[38] H. Belich, F. J. L. Leal, H. L. C. Louzada and M. T. D. Orlando, Phys. Rev. D 86, 125037 (2012).

[39] K. Bakke and H. Belich, Ann. Phys. (N. Y.) 373, 115 (2016).

[40] R. L. L. Vitória and H. Belich, Eur. Phys. J. C 78, 999 (2018).

[41] R. L. L. Vitória, H. Belich and K.Bakke, Adv. High Energy Phys. 2017, 6893084 (2017).

[42] K. Bakke and H. Belich, Ann. Phys. (N. Y.) 360, 596 (2015).

[43] R. L. L. Vitória, K.Bakke and H. Belich, Ann. Phys. (N. Y.) 399, 117 (2018).

[44] R. L. L. Vitória, H. Belich and K.Bakke, Eur. Phys. J. Plus 132, 27 (2017). 
[45] H. F. Mota, K. Bakke and H. belich, Int. J. Mod. Phys. A 32, 1750140 (2017).

[46] F. Ahmed, IJMPA (2021), DOI:10.1142/S0217751X21501281.

[47] F. Ahmed, Sci. Rep. 11, 1742 (2021).

[48] A. Ronveaux, Heun's Differential Equations, Oxford University Press, Oxford ( 1995).

[49] S. Y. Slavyanov and W. Lay, Special Functions: A Unified Theory Based in Singularities, Oxford University Press, New York (2000).

[50] G. B. Arfken and H. J. Weber, Mathematical Methods For Physicists, Elsevier Academic Press, London (2005).

[51] A. F. Nikiforov and V. B. Uvarov, Special functions of mathematical physics (Birkhauser, Basel, 1988).

[52] M. Abramowitz and I. A. Stegum, Hand book of Mathematical Functions, Dover Publications Inc., New York (1965).

[53] F. Ahmed, Eur. Phys. J. C 79, 682 (2019). 